\title{
e-Phaïstos
}

e-Phaïstos

Revue d'histoire des techniques / Journal of the history

of technology

VII-1 | 2019

Le travail humain

\section{Le mobilier de bureau, une mesure déléguée}

office Furniture, a Delegated Measure

\section{Thierry PILLON}

URL : http://journals.openedition.org/ephaistos/4511

DOI : 10.4000/ephaistos.4511

ISSN : 2552-0741

\section{Éditeur}

IHMC - Institut d'histoire moderne et contemporaine (UMR 8066)

\section{Référence électronique}

Thierry PILLON, «Le mobilier de bureau, une mesure déléguée », e-Phaïstos [En ligne], VII-1 | 2019, mis en ligne le 06 avril 2019, consulté le 19 avril 2019. URL : http://journals.openedition.org/ ephaistos/4511 ; DOI : 10.4000/ephaistos.4511

Ce document a été généré automatiquement le 19 avril 2019.

Tous droits réservés 


\section{Le mobilier de bureau, une mesure déléguée}

Office Furniture, a Delegated Measure

Thierry PILLON

\section{L'oubli de l'outil}

Dans son ouvrage de 1926, La technopsychologie du travail industriel, le psychologue Léon Walther distingue l'adaptation de l'ouvrier à son travail, de l'adaptation du travail à l'ouvrier. La sélection professionnelle d'un côté, le procédé et l'outillage de l'autre. Il propose de passer de l'étude des aptitudes psychologiques aux attitudes physiques et de celles-ci à la conformité des outils, élargissant ainsi le point de vue sur le travail concret. La démarche est originale; elle articule les capacités énergétiques et les postures mesurées à l'organisation technique et matérielle du travail.

2 Le chapitre consacré à l'outillage mérite attention. L. Walther constate le peu d'intérêt qu'a suscité l'étude de l'outillage pourtant si nécessaire à la juste compréhension de la dépense énergétique. "Très peu de recherches » dit-il, "ont été faites pour étudier scientifiquement l'outillage dans le sens le plus large du mot $»^{1}$. De nombreux travaux se sont en effet concentrés sur les dépenses énergétiques de l'ouvrier en tenant compte de l'outillage tel qu'il était donné; des recherches centrées sur la bonne attitude plutôt que sur la transformation de l'outil lui-même. C'est par exemple le cas de Jules Amar lorsqu'il évalue la dépense d'énergie - mesurée en oxygène consommé - selon les positions du corps $^{2}$. Ou bien lorsqu'il étudie les postures les plus économes en énergie dépensée dans la manutention d'une brouette, ou encore dans sa célèbre étude sur l'analyse des efforts de respiration du limeur selon « la bonne ou mauvaise attitude pour travailler $»^{3}$.

3 L'originalité du regard de Léon Walther, c'est de saisir le travail dans sa dynamique temporelle, l'adaptation de l'environnement technique à l'usage. L'outil y apparaît comme une extension du mouvement naturel; son ajustement repose sur une définition comme «segment nouveau ajouté à la chaîne des segments du corps humain $»^{4}$. Se 
déploie toute la complexité du rapport ainsi compris. L'outil est considéré sous le point de vue de sa forme, de sa masse, de sa longueur, de son poids, de son volume dont il faut selon les circonstances déterminer les meilleures proportions. Sa construction est à rapporter à son mode d'action sur la matière : «quelle doit être la forme par rapport à la zone d'attaque? $»^{5}$. Quelle longueur doit avoir le manche d'un outil? Sur ces questions nous dit $\mathrm{L}$. Walther, «nous ne possédons presque point de recherche ${ }^{6}$.

Mais, en soi, l'outil n'est rien. Puisqu'il est prolongement du corps, sa position dans l'espace devra se trouver en " accord harmonieux avec le moteur psycho-physiologique de l'ouvrier "; des leviers par exemple, bien positionnés, bien proportionnés n'exigent de l'ouvrier « que les mouvements les plus naturels et les plus économes dans leur parcours dans l'espace $»^{7}$. Enfin, la conception de l'espace de travail devra décharger l'ouvrier de la coûteuse «fonction psychique » d'attention et éviter toute dispersion d'énergie. En 1926, L. Walther fait le constat d'une expérimentation empirique de ces questions. Rien ne permet de définir à l'époque «une sorte de canon qui servirait de base pour la construction de n'importe quel outil ou machine $»^{8}$. La raison tient sans nul doute à la diversité des situations de travail ; elle tient surtout sous la plume de L. Walther à une conception très originale de l'outil comme matière malléable, se modifiant dans l'usage prolongé qu'en fait l'ouvrier'.

\section{Rationaliser le travail administratif}

5 Aussi avancées que soient les propositions de Léon Walther en 1926, elles reposent sur le constat d'un moindre intérêt pour les questions d'ergonomie, d'adaptation de l'outillage aux nécessités du travail. Diversité des outils, des situations, centration sur la mesure de l'énergie dépensée et celle des aptitudes: l'outil a été en grande part délaissé. Il a rarement été compris comme une ressource, facteur du rendement recherché presque exclusivement dans l'économie psycho-physiologique, à l'exception du bureau peut-être ${ }^{10}$ . Car l'intérêt qu'on porte au mobilier dès le début du $\mathrm{XX}^{\mathrm{e}}$ siècle, et surtout entre les deux guerres, nous permet de déplacer le regard vers les moyens mis en œuvre pour réaliser le programme des sciences du travail : rationaliser le geste, augmenter le "rendement", diminuer la fatigue. Ces objectifs s'inscrivent dans le cadre d'une science du travail administratif imposée par le développement des activités tertiaires au début du $\mathrm{XX}^{\mathrm{e}}$ siècle. Inspiré du modèle taylorien américain, puis de celui français de Fayol, la rationalisation du travail de bureau s'appliquera à rompre avec les méthodes du XIX ${ }^{\mathrm{e}}$ siècle : inertie, lenteur, incohérence. L'adoption des "méthodes modernes en affaires » selon le titre d'un ouvrage de 1919, considère désormais la possibilité d'organiser "scientifiquement" les bureaux ${ }^{11}$. L'entreprise y est vue comme une "organisation" traversée d'un mouvement de circulation continue d'informations. La production et le classement de celles-ci, sous forme de fiches, nécessiteront une machinerie et un mobilier original, outils principaux de l'efficacité du travail ${ }^{12}$. Des ouvrages, des revues voient le jour et soutiennent un tel projet. Il est par exemple clairement exprimé au tout début du $\mathrm{XX}^{\mathrm{e}}$ siècle dans la revue Mon bureau, première revue française consacrée au travail administratif: «La routine, l'inertie, l'obstination font piétiner sur place un grand nombre d'organisations, et, cependant, l'effort est aussi grand, souvent même plus grand pour obtenir ce résultat négatif. Les méthodes progressives consistent surtout à se débarrasser de la routine, à créer une organisation qui utilise avec avantage toutes les forces disponibles, à rendre chaque mouvement productif, à éviter la répétition inutile du 
même travail, à supprimer si possible, mais diminuer en tous cas, les courses du personnel d'un bureau, d'un étage, d'un atelier à un autre, à donner de la flexibilité, de la souplesse à cette organisation pour qu'elle se prête sans effort, presque naturellement aux transformations, à l'évolution permanente de l'état social ou commercial avec lesquelles elle doit compter pour ne pas marquer le pas $»^{13}$. Supprimer les mouvements inutiles, majorer l'usage des forces, augmenter leur productivité, favoriser la flexibilité, supprimer les efforts : autant d'ambitions qui sont ici déléguées en grande partie à la conception du mobilier et à l'organisation des espaces. La puissance d'action et ce qu'elle recèle de rendement ne viendront pas donc du corps seul, de ses capacités énergétiques, de la régulation de son effort, de ses aptitudes mesurées en laboratoire, mais d'une écologie propre au bureau fait d'harmonie entre l'outillage et l'usager.

\section{Le tiroir du bureau}

La recherche d'une économie de mouvement dans l'enchaînement ordonné des gestes repose sur des inventions très simples : la fermeture des tiroirs par exemple. Comment produire un effet démultiplié avec un seul geste? En offrant la solution technique d'un seul tiroir à fermer qui bloquera tous les autres. Une telle solution suppose l'existence de tiroirs superposés dont la fermeture les uns après les autres a pu sembler trop longue, source inutile de fatigue, sans pour autant que celle-ci soit clairement mesurée. Ce qu'exige le tiroir, c'est de protéger les documents qu'il renferme et d'être manipulé sans effort. Ce que propose par exemple le brevet d'invention $\mathrm{N}^{\circ} 370.556$ du 13 octobre 1906 : « Perfectionnement dans la fermeture simultanée d'une série de tiroirs ou d'objets analogues: (...) perfectionnements qui (...) permettent de bloquer simultanément la totalité de ces tiroirs en fermant un seul d'entre eux ». Le tiroir fait l'objet d'un intérêt particulier puisqu'il recèle le secret du travail administratif et des affaires: la multiplicité des fiches dont l'ordonnancement et le rangement deviennent rapidement l'objet d'une véritable science. Manipulé à tout moment, le tiroir du meuble à fiches doit pouvoir répondre sans effort. L'usage de galets et de roulements sur des meubles d'abord en bois puis en métal facilite ainsi le déplacement du caisson. En 1919, à propos des meubles classeurs en acier, on remarque qu'ils "séduisent beaucoup par leur parfait glissement; ils sont, en effet, établis sur galets de roulements de manière que la manœuvre des tiroirs assez lourds, étant donné leur contenu, se fasse rapidement et sans effort : le classement étant très souvent fait par des femmes ou des jeunes filles $»^{14}$. Afin d'éviter tout risque de basculement du tiroir: "On a imaginé également des tampons d'arrêt pour obvier à l'inconvénient et au danger d'une manœuvre trop brusque $»^{15}$. Le meuble assiste le geste, et plus encore amplifie ses capacités d'action. Ce nouveau mobilier témoigne d'une rationalité nouvelle du travail de bureau; dans l'efficience accordée aux mouvements les plus élémentaires, mais aussi les plus répétés, se trouve le ressort d'une économie de la fatigue en quelque sorte confirmée par l'usage. Car dans les brevets comme dans la description qu'en donne la littérature spécialisée, il n'est pas question d'un calcul précis de la dépense que demande, par exemple, l'ouverture d'un tiroir. Si on rapporte bien le matériel à l'effort, c'est le plus souvent en référence à une évaluation empirique. Celle-ci renvoie aux anciens mobiliers si difficiles à manipuler; mais surtout à l'usage que peuvent en faire les femmes, catégorie d'employées désignées comme physiquement la plus faible. Ainsi, entre les deux guerres à propos d'un meuble de classement, on souligne sa facilité d'usage avec cette même approximation : «L'emploi de classier qui ne demande 
pas d'efforts musculaires exagérés, si le matériel est de bonne qualité, peut convenir parfaitement à une femme très soigneuse, intelligente et minutieuse. Il demande plus de soins et de conscience que d'énergie et d'initiative $»^{16}$. Même si, pour le même meuble, l'auteur souligne qu'il ne « dépasse pas 1,30 mètre (et) permet l'accessibilité rapide à tous les tiroirs ", la question essentielle reste cette commodité d'usage simplement exprimée comme "moindre effort». La délégation des tâches intellectuelles aux différents systèmes de classement et de calcul, dont D. Gardey a finement étudié le développement en France à cette période ${ }^{17}$, n'est pas dissociable d'une recherche conjointe d'un mobilier économe en énergie, en fatigue, et adapté à la distribution inégale des capacités physiques des employés.

\section{Le bureau}

7 Si le tiroir s'avère être un élément plus technique qu'il n'y paraît - il sera par exemple muni de compresseurs à crémaillères qui maintiennent les dossiers rassemblés - c'est également le cas du bureau. Dès le début du $\mathrm{XX}^{\mathrm{e}}$ siècle, il se transforme et acquiert un nouveau statut. De simple meuble, souvent issu du monde domestique, il devient outillage. Le constat est fait par le technicien-conseil Marc Grandjean en 1921 dans la revue Mon bureau ${ }^{18}$. À cet égard, il répond en grande partie aux exigences que Léon Walther assignait à l'adaptation des outils industriels au travail ouvrier : enchaîner des opérations sans effort, économiser les mouvements et la dépense d'attention. Dans les bureaux, nombre d'inventions témoignent d'un souci d'adapter le meuble aux besoins et à la diversité des postes de travail. D'une manière générale, il s'agit toujours de prévenir l'encombrement, d'économiser le temps de classement et de rangement, en somme, d'optimiser le travail de l'employé. Au tout début du siècle, les solutions techniques passent le plus souvent par la mécanisation du meuble. C'est le cas par exemple du Meuble-bureau à deux tables de travail ${ }^{19}$ qui permet de travailler sur deux tables de travail simultanément, bien que sur des plans différents. L'inventeur décrit ainsi son projet: « L'invention consiste essentiellement en un meuble bureau qui, au moyen du mécanisme employé, peut prendre différentes positions, lesquelles sont la conséquence d'un triple mouvement combiné par suite duquel les deux tables de travail dont le bureau est formé reculent, avancent et montent sans qu'il soit nécessaire d'en retirer les papiers documents, etc. ». Les transformations qu'offre le meuble sont destinées à répondre à toutes les possibilités de travail envisageables, même si concrètement la manipulation du bureau lui-même peut s'avérer complexe. C'est le cas également du Bureau à transformation « dont une partie peut se déplacer verticalement de manière à donner au bureau, quand cette partie est rentrée à l'intérieur, l'apparence d'un bureau ministre, et de lui laisser en même temps les avantages des bureaux américains au point de vue du nombre des tiroirs et compartiments $»^{20}$. Il s'agit bien de rompre avec les vieux meubles, mal conçus, mal adaptés. Mais surtout d'épouser l'ensemble des mouvements que doivent exécuter les employés. Pour une secrétaire, par exemple, dès lors que son activité s'est étendue avec la complexification du travail administratif, il lui faut un outil qui puisse y répondre en tout point : «on s'est aperçu qu'une secrétaire ne fait pas que taper sur la machine à écrire : elle classe les fiches, écrit sur certains registres, a besoin, en fait, d'une table à écrire ». Le bureau doit proposer un tout-en-un : « Une table à écrire assez grande (115x70) sur le côté une planchette articulée ad libitum portant la machine à écrire, et rentrant avec celle-ci sous le bureau. Les papiers sont rangés dans un casier à droite et 
sur le bureau. Il n'échappe à personne que la dactylographe a, devant elle, une surface toujours en ordre : un véritable bureau $\aleph^{21}$. Système de renversement, disparition de la machine dans le corps du meuble, tablette en mouvement, si le bureau est devenu un outil, c'est aussi qu'il s'harmonise avec les nécessités qu'imposent les tâches précises et plus encore avec l'organisation particulière de chacune des entreprises dans le but, toujours, de "réduire l'effort de chacun au minimum ${ }^{22}$. Comme le remarque Albert Navare en 1924, « Pour produire le maximum de bon travail avec le minimum de fatigue, les dactylographes doivent être installés confortablement (ce qui n'est pas ici synonyme de luxe), avoir notamment à leur portée les accessoires dont ils ont fréquemment besoin (...) $)^{23}$. Le confort en question ne se déduit pas pour autant de canons anthropométriques. Quand il s'agit de préciser les formats du bureau, l'énoncé des chiffres s'inspire de l'expérience de l'usager. Pour le bureau de secrétaire considéré comme outil moderne, Marc Grandjean note que « la tablette de la machine à écrire doit être à 63-65 centimètres au-dessus du sol. La hauteur du bureau étant de $78 \mathrm{~cm}$, c'est donc un décalage de 13 à $15 \mathrm{~cm}$. Le porte à faux doit être au moins de $50 \mathrm{~cm}$. Si la machine à écrire était trop près du bureau, le coude gauche de l'opérateur serait gêné ${ }^{24}$. De même, lorsqu'il s'agit de savoir, en 1924, quelle est la bonne hauteur de table pour supporter la machine à écrire, elle ne doit être "ni trop haute, ni trop basse. On en trouve dans le commerce de parfaitement appropriées. La dimension généralement adoptée est de 60 centimètres de haut, soit de 10 à 15 centimètres plus bas que les tables ordinaires ${ }^{25}$. À mesure que se complexifie le travail de l'employé(e), ici de la dactylo, le meuble déploie donc de nouveaux « organes » pour y répondre ; de la simple table sur laquelle on a posé une machine, le bureau devient un "collaborateur vivant» et souple. C'est de cette collaboration harmonieuse qu'est attendue l'économie de fatigue, la réduction de "l'effort de chacun au minimum ${ }^{26}$. Au cours des années 1930, le meuble-outil en acier répond aux mêmes intentions que le bureau en bois: «utile et logique (...), il est nécessaire que cet instrument réponde parfaitement et uniquement à l'usage qu'on attend de lui, qu'il ne mente pas, qu'il n'ait rien en lui pour tromper, retarder, enrayer une activité. Au contraire, un bureau destiné par définition à un usage déterminé encourage "; un outillage donc qui « continue nos mouvements, qui est conforme à notre corps, participe à une certaine harmonie, au même titre que d'autres accessoires » ${ }^{27}$.

\section{L'émergence d'une géométrie}

8 Un des principes de cette harmonie est celui de l'extension donnée au bras : avoir tout à portée de main limite la dispersion de l'attention. Le brevet d'invention du Bureau calculateur en 1919 souligne ainsi que «La présente invention, a pour objet, un procédé qui consiste à placer sur la tablette d'un bureau, sans gêner en rien l'usage de cette dernière, sur les parties les plus à la portée de l'œeil et de la main de la personne travaillant au bureau: les moyens de calculs rapides demandés par chacun des acheteurs, ainsi qu'une machine à calculer susceptible de s'encastrer dans ou sous la tablette du bureau, sans produire aucune saillie sur cette dernière ${ }^{28}$. La même année 1919, avec le "Bureau directorial » présenté dans l'ouvrage de Jules Lepain et Jacques Granville, l'emplacement des tiroirs permet à l'usager d'avoir toutes ses fiches classées "à portée de la main ", «sans quitter son fauteuil»; pour accéder aux informations, "point besoin de se déplacer pour aller à une bibliothèque. Nous n'avons qu'à étendre la main et ouvrir un tiroir $»^{29}$. La main acquiert ainsi une capacité d'action nouvelle qui n'est plus seulement 
celle de l'écriture. L'ensemble des équipements techniques en décuple la puissance et lui confère un champ d'action plus opérant. "Étendre la main ", ou saisir une information d'un seul regard, participe à la rationalisation du travail administratif en devenant la source nouvelle d'un rendement présenté sous le registre du confort. Le bureau est alors d'autant plus conçu comme outillage performant qu'il s'articule aux machines de bureau dont la capacité à traiter rapidement de l'information complexe passe par leur qualité ergonomique. L'entreprise française "Le calcul mécanique» présente en 1924 une machine permettant d'avoir également, « Tout sous les yeux, tout sous les doigts ${ }^{30}$.

Si le geste accroit son rendement, au bénéfice d'une efficience plus grande de chacun des mouvements dirigés, il ne fait que rarement l'objet d'une mesure précise. Du moins en France, dans les années 1900-1920, chez les promoteurs de l'organisation rationnelle du bureau. Car, aux États-Unis, à la suite des travaux de F. Gilbreth ${ }^{31}$ repris par Ralph Barnes ${ }^{32}$, les recherches sur l'amplitude et les temps de déplacement des membres supérieurs détermineront plus précisément la géométrie des zones de travail. En France, la présentation au Salon des Artistes décorateurs en 1934 du «Bureau rationnel » dessiné par l'architecte Maurice Barret s'inscrit dans cette perspective, bien qu'aucune précision concernant les recherches citées ne soit apportée: «(...) nous avons conclu après ces premières recherches que l'utilisation de deux plans à angle droit avec le siège pivotant permettait de donner au bureau une forme nouvelle et réellement efficiente. Par surcroit, cette forme s'adapte bien au fonctionnement de nos bras qui n'agissent pas seulement devant eux, mais se déplacent naturellement par côté. (...) Cet aménagement général, qui participe d'une étude technique de l'économie des gestes, du confort rationnel (...) $»^{33}$. Dès la fin des années 1930, Ralph Barnes donnera quant à lui des indications précises sur les zones de travail et l'économie de fatigue qu'elles permettent. Ainsi, La zone normale de travail : « de la main est déterminée par un arc de cercle tracé en balayant de la main le poste de travail ; l'avant-bras seul est étendu, le bras pend naturellement le long du corps jusqu'à ce qu'il tende à s'écarter quand la main s'éloigne de la surface du travail. Les deux axes tracés par chaque main se coupent en un point situé devant l'opérateur ${ }^{34} »$. Cette première zone est fonction de la " dépense d'énergie normale ». La zone maximale de travail est celle au contraire « en dehors de laquelle on ne saurait accomplir de travail à deux mains sans modifier notablement la position du corps, modification qui s'accompagne d'une fatigue excessive $»^{35}$. Cette géométrisation des gestes s'accomplit pleinement dans la suppression de tout effort mental. L'exacte mesure de leurs amplitudes permet aux mains de se déplacer sans effort: «L'exécutant ne doit pas avoir à réfléchir pour savoir où prendre les matériaux » et "Accomplir son travail avec un minimum d'effort mental conscient $\aleph^{36}$, c'est-à-dire avec toute l'automaticité que permet le geste rapide sans le recours de la vue, encore trop coûteux en temps et en attention ${ }^{37}$. Les profondeurs normalisées des surfaces de bureaux et des tables de travail découleront de ces mesures ${ }^{38}$

10 Le meuble bureau-outil apparait alors à la fois comme "puissance de concentration ", ${ }^{39}$ puisque son organisation condense les informations contenues dans ses tiroirs où sont rangées les fiches et les dossiers selon une disposition rationnelle qui permet de les retrouver facilement et sans effort ; mais il est également le prolongement naturel de nos membres, puisqu'il offre à portée de main tous les outillages et matériels utiles au déroulement continu du travail. Ces compétences objectives inscrites dans l'artefact technique sont très clairement exprimées par André Beucler lorsqu'il fait la promotion du meuble en acier en $1930:$ : (...) l'ameublement métallique du bureau moderne dirige vers 
leur accomplissement parfait certaines positions du corps : celle de s'asseoir, de classer ; certains désirs : le besoin d'éviter la fatigue, d'avoir l'impression de l'aisance, de trouver autour de soi des objets types et de savoir que le travail est plus facile $»^{40}$. C'est dans des termes proches qu'en 1925 Le Corbusier décrivait déjà les mobiliers métalliques de la marque Ronéo. Ils viennent à la fois "compléter» et renforcer nos "capacités naturelles » et répondre à nos besoins; "cette mécanique nous entourant (...) n'est exactement que le prolongement de nos membres; ce sont à vrai dire des membres artificiels » dont le but est de nous éviter de la fatigue et de « libérer notre esprit » ${ }^{41}$.

\section{Chaises et fauteuils}

11 La fluidité qu'autorise le bureau-outil n'est complète que si le fauteuil ou la chaise ne la limite pas. La chaise sur roulettes et à hauteur modulable, ainsi que le fauteuil, dont les premiers modèles sont américains ${ }^{42}$, jouent à cet égard un rôle essentiel. Au début du XX siècle en France, ils offrent des hauteurs réglables permettant de s'adapter « aux tables dont la hauteur doit être variée $»^{43}$; mais également « suivant la taille de la personne qui voudra s'asseoir $»^{44}$. À la hauteur s'ajoute l'inclinaison variable qui donnera lieu à de nombreux sièges et chaises longues ${ }^{45}$. Le fauteuil doit épouser naturellement tous les mouvements du corps exigés par les tâches professionnelles. Pour les promoteurs du bureau moderne, la référence est explicitement américaine. Au fauteuil ordinaire qui " enferme » l'utilisateur et le condamne à l'immobilité, l'oblige sans cesse à se lever pour atteindre ses documents, recevoir un visiteur, se substitue un principe dynamique incarné dans les modèles américains : «La nécessité s'imposait donc absolument, autant pour l'aise du travail que pour l'économie du temps, de créer un meuble spécial capable de se prêter à tous les mouvements sans que celui qui y est assis ait à l'abandonner. Vous ne doutez point que la difficulté ait été résolue. Elle le fut, et promptement en effet, par la création du fauteuil basculant et tournant qui est d'invention franchement américaine $»^{46}$ . Ce sont donc tous les mouvements possibles que le fauteuil doit pouvoir accompagner, sans effort particulier. Les crémaillères permettent d'abaisser et de relever le siège en fonction de la taille de l'utilisateur, un pivot permet le basculement d'avant en arrière, la " combinaison de ces deux mouvements assure la possibilité de tous les déplacements imaginables sans abandonner son siège $\aleph^{47}$. Et c'est bien la fatigue qui est visée. Par la mobilité qu'il permet, le siège constitue avec le bureau un autre principe d'économie de dépense : «De cette large aisance de mouvement résulte pour le négociant une épargne précieuse de temps et de fatigue $»^{48}$. La chaise de dactylo créée par l'ingénieur Henri Liber à la fin des années 1920 illustre mieux que d'autres cette ambition ${ }^{49}$. Si elle reprend tous les principes dynamiques des chaises américaines, elle y ajoute un réglage en hauteur du dossier selon la taille de l'utilisateur et la possibilité de déplacements latéraux du corps. L'assise épouse les mouvements de la secrétaire si celle-ci se penche sur le côté. Des ressorts permettent un réglage de la «flexibilité » du dossier. Le modèle, dit une publicité, est « flexible dans tous les sens ». En 1926, la chaise se fixe à un rail par ses deux pieds avant, les deux autres sur roulettes. Le système permet ainsi « à la personne assise sur le siège de se déplacer devant une table, un meuble classeur, un fichier, un établi, etc., sans aucun effort et sans avoir besoin de se soulever " ${ }^{50}$. Il suffira en effet de déplacer légèrement le poids du corps en avant pour permettre le mouvement latéral le long du rail. Un an plus tard, Henri Liber dépose un nouveau brevet permettant de faciliter la bascule avant du siège par un «ressort à boudin» et fixe sur le dossier un coussin en 
"matière élastique, caoutchouc mousse ${ }^{51}$. La chaise d'Henri Liber sera présentée à l'exposition internationale de Paris de 1937 comme témoignage de l'avancée française en matière d'équipement de bureau.

\section{Conclusion}

Déplacer le regard vers le meuble, c'est concevoir autrement les sources de l'efficacité du travail. Le souci du rendement et de rationalité qui caractérise le monde du bureau au début du XXe siècle et entre les deux guerres fait reposer son exigence sur une rhétorique du confort, c'est-à-dire une prise en considération par les meubles eux-mêmes d'une économie corporelle naturelle : fluidité des déplacements, extension du rayon d'action des membres, enchainement des gestes simples. L'efficience et l'épargne de fatigue sont donc en partie concédées aux caractéristiques techniques du mobilier à travers l'équilibre des énergies qu'il parvient à réaliser entre le corps et son milieu. Mais " collaborateur vivant", le bureau l'est aussi en ce qu'il impose des manières de faire et des usages particuliers. Son adaptation au corps a aussi une dimension prescriptive, formatrice d'habitude, ce qui n'est pas un des moindres ressorts de cette économie de la fatigue. Il impose de nouveaux rythmes au travail, permet des mouvements continus, des enchaînements sans repos; une fluidité vantée par les organisateurs qu'il faut aussi comprendre comme la recherche d'un avancement sans faille du travail. En ce sens, le meuble - ici le bureau - condense dans la perfection de sa conception une nouvelle norme de travail tertiaire, une rationalité déléguée en quelque sorte à l'objet toujours prompt à répondre à toute nécessité imposée par l'exigence de l'activité en cours.

\section{NOTES}

1. WALTHER Léon, La technopsychologie du travail industriel, Neuchâtel, Paris, Delachaux \& Nieslé, 1926, p.152

2. AMAR Jules, Le moteur humain et les bases scientifiques du travail professionnel, Paris, Dunot et Pinat, 1913, p.445.

3. AMAR Jules, Le travail humain, Paris, Librairie Plon, 1923, p.56. D'une manière générale, les «sciences du travail» se sont peu préoccupées de la question des outils, du matériel, de l'organisation de l'espace de travail. Voir, par exemple, pour une présentation d'ensemble de ces sciences du travail, RABINBACH Anson, Le moteur humain. L'énergie, la fatigue et les origines de la modernité, Paris, La Fabrique édition, 2004 ; SARACENO Marco, Pourquoi les hommes se fatiguent-ils. Une histoire des sciences du travail (1890-1920), Toulouse, Octarès, 2018.

4. WALTHER Léon, La technopsychologie..., op.cit. 1926, p.157.

5. WALTHER Léon, Ibidem, p.158. L'interprétation d'une interaction entre matériaux et action mécanique sera développée dix ans plus tard dans l'article que Leroi-Gourhan rédige pour L'encyclopédie française, en 1936, dans lequel l'essentiel de sa classification future est déjà présente. Voir la présentation qu'en fait Sophie de BEAUNE dans «La genèse de la technologie comparée chez André Leroi-Gourhan. Introduction à son article 'L'homme et la nature'", 
Documents pour l'histoire des techniques, Ibis Press et Centre d'Histoire des Techniques CNAMEHESS, 2011, p.197-223. <halshs-00730327>.

6. WALTHER Léon, La technopsychologie..., op.cit. 1926, p.161.

7. Ibidem, p.162.

8. Ibid., p.158.

9. Plutôt que de se fier à l'instinct de l'ouvrier, dit-il, « il paraît plus juste de suivre de près et d'étudier la forme que l'outil a pris par soi-même après de longues années d'usage, grâce à sa forme plus ou moins élastique ». L. Walther donne ainsi l'exemple d'un marteau « qui après vingt et un ans, s'est complétement adapté à la main de l'ouvrier ». La méthode est singulière : « elle consiste à expérimenter avec des matières élastiques qui, maniées quelque temps par des ouvriers habiles, prendront la figure la plus conforme à la peau et à la musculature ». C'est ainsi, selon une étude allemande citée par L. Walther, qu'est déterminée la forme adéquate d'une manivelle de conducteur de tramway. Ibid., p.158-159.

10. Il ne s'agit pas d'oublier les travaux psychophysiologiques ou psychotechnique sur le travail des employés, des dactylos en particulier, mais rien n'est dit sur leur environnement de travail, la disposition de leur bureau, l'usage qu'elles peuvent en faire et qu'elle est la fonction du meuble dans l'économie générale de la fatigue, ou les capacités d'apprentissage. Voir, par exemple, les travaux de LAHY Jean-Maurice, «Le facteur psychologique dans la construction des machines à écrire ", L'année psychologique, 1927, vol.28, p.245-247 ; LAHY Jean-Maurice, KORNGOLD S.V., «Sélection des opératrices de machines comptable», L'année psychologique, 1931, vol.32, p.131-149.

11. LEPAIN Jules, GRANDVILLE Jacques, Les méthodes modernes en affaires. La psychologie dans les affaires. L'organisation scientifique des bureaux et magasins, Paris, Nouvelle librairie nationale, 1919.

12. GARDEY Delphine, La dactylographe et l'expéditionnaire, histoire des employés de bureau (1890-1930) , Paris, Belin, 2004.

13. Mon bureau..., op. cit., 1909 , p. 56

14. LEPAIN Jules, GRANDVILLE Jacques, Les méthodes modernes..., op.cit., 1919, p.149-150.

15. Ibidem.

16. RAVISSE Gaston, « La technique des classements », dans RAVISSE Gaston (dir.), La technique du travail de bureau, Paris, Librairie française de la documentation commerciale \& industrielle, 1928, p.88.

17. GARDEY Delphine, Écrire, calculer, classer. Comment une révolution de papier a transformé les sociétés contemporaines (1800-1940), Paris, La Découverte, 2008.

18. GRANDJEAN Marc, "Le meuble de bureau est-il un outillage?», Mon bureau, mars 1921, p.185-186.

19. Office National de la Propriété Industrielle, Brevet d'invention $N^{\circ} 431.207$ du 16 juin 1911, «IX - Matériel de l'économie domestique - 4 Meubles et ameublement, mobilier des jardins ».

20. Office National de la Propriété Industrielle, Brevet d'invention $N^{\circ} 433.561$ du 23 août 1911. « IX - Matériel de l'économie domestique - 4 Meubles et ameublement, mobilier des jardins ».

21. GRANDJEAN Marc, « Le meuble... », op.cit., 1921 p. 186

22. « Le bureau moderne ", Mon bureau, novembre 1913, anonyme, p.705.

23. NAVARRE Albert, Manuel d'organisation du bureau, Paris, Librairie Delagrave, 1924, p.46. A. Navarre parle ici de dactylographe au masculin, la profession conserve encore à cette époque une population d'employés dactylographes hommes. Sur ce point, voir GARDEY Delphine, « Du veston au bas de soie, identité et évolution du groupe des employés de bureau (1890-1930) ", Le mouvement social, $\mathrm{n}^{\circ} 175$, avril-juin 1996, p.53-75.

24. GRANDJEAN Marc, « Le meuble... », op.cit., 1921, p.186.

25. NAVARRE Albert, Manuel..., op.cit., 1924, p.46.

26. « Le bureau moderne », op.cit., 1913, p.705. 
27. BEUCLER André, « Meubles métalliques », Acier, n¹, 1930 (sans pagination), Publié par l'office technique pour l'utilisation de l'acier. La standardisation n'est pas absente d'un tel processus de transformation du meuble. La largeur des tiroirs dépendra de celle des fiches, elles-mêmes du papier à lettre correspondant aux largeurs des machines à écrire; les classeurs verticaux pourront ensuite être assemblés par éléments jusqu'à composer des murs entiers de rangements standardisés. L'utilisation de l'acier dans la fabrication de ces systèmes de rangements dans les années 1930 permettra d'atteindre des surfaces considérables, de diminuer les encombrements et de se prémunir des risques d'incendie. Les fabricants de meubles en acier devront répondre aux exigences de simplicité et de facilité d'usage des bureaux et des systèmes de rangement. Plusieurs entreprises se spécialisent à cette époque dans le mobilier administratifs et domestique en acier : Roneo; Les meubles superposables, Strafor; Flambo; Meubles Mulpitl's. Des entreprises métallurgiques fabriqueront leur propre mobilier, c'est le cas des cycles Peugeot qui fabrique du mobilier en tube pour équiper ses bureaux en 1935.

28. Office National de la Propriété Industrielle, Brevet d'invention n506.089 du 6 novembre 1919. «IX - Matériel de l'économie domestique - 4 Meubles et ameublement, mobilier des jardins ». C'est nous qui soulignons.

29. LEPAIN Jules, GRANDVILLE Jacques, Les méthodes modernes..., op.cit., 1919, p.148.

30. Publicité dans Mon bureau, op. cit., décembre 1924, p.353.

31. Le livre de GILBRETH, F.B., Motion Study, est publié en 1911 et traduit en 1921 sous le titre, Étude des mouvements appliqués. Série de mémoires concernant la méthode d'organisation à employer dans l'industrie, Paris, Dunod, 1921

32. BARNES Ralph, «Étude du temps et des mouvements à l'aide du cinéma », Revue internationale du cinéma éducateur, $\mathrm{n}^{\circ} 6,1933$.

33. Mon bureau, juillet-août 1934, p.348.

34. BARNES Ralph, « Étude du temps... », op.cit. 1949, p.237-238.

35. Ibidem, p.239.

36. Ibid., p.234.

37. On notera qu'en France, ces recherches sur la disposition des espaces de travail proche, seront poursuivis après la Seconde Guerre mondiale, par exemple dans la thèse du professeur de physiologie et ingénieur en O.S.T., BOUISSET Simon, au CNAM en 1957, Utilisation par l'Organisation scientifique du Travail des études relatives aux gestes libres, gestes asservis, gestes directeurs. Dans ses articles pour Le travail humain, ou à la suite des travaux américains, il explore les meilleures dispositions spatiales des surfaces de travail dans la perspective d'une économie de mouvement combinée à une efficacité du geste, BOUISSET Simon, "Problème posé par l'aménagement des postes de travail : les règles d'économie des mouvements ", Le travail humain, Vol. 25, juil-déc. 1962, p.281-304.

38. Voir par exemple BARNES Ralph, «Étude du temps... », op.cit. 1949, p.238, 266 et 279.

39. LEPAIN Jules, GRANDVILLE Jacques, Les méthodes modernes..., op.cit., 1919, p. 149.

40. BEUCLER André, « Meubles métalliques », op.cit., 1930.

41. LE CORBUSIER, L'art décoratif aujourd'hui (1925), Paris, éditions Flammarion, 1996, p.72-75. En 1938, Charlotte Perriand dessine pour Jean-Richard Bloch, le directeur du journal Ce Soir, un «Bureau en forme » dont la géométrie offre à l'utilisateur un rayon d'action optimum.

42. GIEDION Sigfried, La mécanisation au pouvoir (1948), Paris, Centre Georges Pompidou, 1980.

43. Office National de la Propriété Industrielle, Brevet d'invention du 9 décembre 1905, N³60.288 Chaise avec siège réglable en hauteur: Chaise à crémaillère. «IX - Matériel de l'économie domestique -4 Meubles et ameublement, mobilier des jardins ».

44. Office National de la Propriété Industrielle, Brevet d'invention du $1^{\mathrm{er}}$ octobre $1902, \mathrm{~N}^{\circ}$ 324.887 : Siège mobile. «IX - Matériel de l'économie domestique - 4 Meubles et ameublement, mobilier des jardins ». 
45. Les mobiliers de bateau, de chemin de fer, ainsi que les meubles professionnels des dentistes et coiffeurs ont été parmi les premiers à proposer des inclinaisons variables. Voir les exemples que donne GIEDON Siegfried, op. cit., et, pour la France du XX $\mathrm{X}^{\mathrm{e}}$ siècle, à titre d'exemple : Brevet d'invention du 15 novembre 1905, N³59.470, Chaise à inclinaisons variables : «Cette invention est relative aux chaises à inclinaisons variables destinées à être utilisées à bord des paquebots et a pour but de construire une chaise simple pouvant être ajustée rapidement de manière à pouvoir supporter une personne dans la position verticale ou dans des positions inclinées variables ».

46. HELLER L.R., Organisation commerciale. Le bureau moderne. Son agencement, son outillage, Paris, éditions de « Mon bureau », 1916, p.27.

47. Ibidem, p.29.

48. Ibid.

49. Henri Liber sera le fondateur de la société Flambo Diffusion en 1930, après l'inauguration d'une usine à Vierzon dans le Cher en 1968. L'entreprise sera liquidée en 1986. Henri Liber collaborera avec les architectes du mouvement moderne, Robert Mallet-Stevens et Charlotte Perriand.

50. Ministère du commerce et de l'industrie. Direction de la propriété industrielle, Brevet d'invention du $1^{\mathrm{er}}$ octobre 1926, N 611.600 , Siège coulissant à déplacement latéral. Un tel système, le « Roule-Dactyle » sera installé au service de la conservation des titres de la Banque Nationale de Crédit en 1931.

51. Ministère du commerce et de l'industrie. Direction de la propriété industrielle, Brevet d'invention du $1^{\mathrm{er}}$ octobre $1926, \mathrm{~N}^{\circ} 33.452,1^{\mathrm{re}}$ addition au brevet Siège coulissant à déplacement latéral.

\section{RÉSUMÉS}

Le meuble de bureau tel qu'il est conçu au début du XXe siècle cherche à rompre avec les modèles anciens de mobiliers inconfortables. Il devient un outil du travail administratif et contribue à réduire les efforts pour rendre plus efficace le travail de l'employé(e) de bureau. Le bureau, la chaise deviennent ainsi les supports d'une rationalisation du travail tertiaire. Celle-ci vise tout autant l'économie de la fatigue que l'efficience requise par la diversité des activités. Ces changements techniques reposent sur des petites inventions parfois discrètes qui ne relèvent pas nécessairement du registre du calcul et de la mesure des capacités physiologiques. L'ergonomie du bureau au début du XXe siècle cherche à concevoir une harmonie entre le corps et son environnement fondée sur la fluidité naturelle des gestes plus que sur la mesure précise de la dépense d'énergie.

The office furniture as it was designed in the early 20th century seeks to break with old models of uncomfortable furniture. It becomes a tool of administrative work and helps reduce efforts to make the work of the clerical employee more efficient. The office and the chair become the supports for a rationalization of tertiary work. It is equally aimed at the economy of fatigue and the efficiency required by the diversity of activities. These technical changes are based on small, sometimes discrete, inventions that are not necessarily related to the register of calculation and the measurement of physiological capacities. The ergonomics of the office in the early 20th century seeks to design a harmony between the body and its environment based on the natural fluidity of the gestures more than on the precise measurement of energy expenditure. 


\section{INDEX}

Mots-clés : histoire des techniques, mobilier, bureau, administration, ergonomie, géométrie, XXe siècle, France, Etats-Unis

Index chronologique : Époque contemporaine

Index géographique : France, USA

Keywords : history of technology, furniture, offices, administrative work, ergonomics, geometry, early 20th century, France, United States

\section{AUTEUR}

\section{THIERRY PILLON}

Professeur de sociologie à l'université Paris 1 Panthéon-Sorbonne, Tierry Pillon est directeur du CETCOPRA, un laboratoire de socio-anthropologie des techniques. Ses principaux axes de recherche sont la sociologie du travail, l'histoire du travail et plus particulièrement ces dernières années la socio-histoire des bureaux sous le registre des relations entre le corps et l'environnement technique. Il s'agit de saisir la question technique à partir des engagements physiques et psychique qu'elle requiert et des perceptions sensibles qu'elle sollicite chez les utilisateurs. Il a publié récemment sur ce thème de recherche : « Les couleurs d'ambiance, L'exemple des bureaux dans les années 1950-1960 ", Communications, n¹02, Paris Seuil, 2018, p.199-208 ; "Von der Hygiene zum Komfort. Das Büro in Frankreich von 1909 bis 1939", in G. Bernasconi, T. Hengartner, A. Kellerhals (ed.), Das Büro : Zur Rationalisierung des Interieurs 1880-1960 , Universität Zürich edit., Zürich, 2019 (sous presse ). Il prépare un travail plus ample sur l'histoire des environnements de travail de bureau. 